\title{
In vitro Antagonistic Mechanisms of Trichoderma spp. and Talaromyces flavus to Control Gaeumannomyces graminis var. tritici the Causal Agent of Wheat Take-all Disease
}

\author{
Leila Ghanbari, Seddighe Mohammadi* \\ Department of Plant Protection, Shiraz Islamic Azad University, Shiraz Branch, Shiraz, Iran
}

\section{A R T I C L E I N F O}

Article history:

Received 13 November 2014

Accepted 23 June 2015

Available online, ISSN: 2148-127X

Keywords:

Colonization

Direct coincidence

Dual culture

Volatile compounds

Non-volatile compounds

\begin{abstract}
A B S T R A C T
Wheat take-all disease caused by Gaeumannomyces graminis var. tritici has recently been detected in different regions of Iran. With respect to biocontrol effect of Trichoderma spp. on many pathogenic fungi, seven isolates of Trichoderma and four isolates of Talaromyces were in vitro evaluated in terms of their biological control against the disease causal agent. In dual culture test the five isolates showed efficient competition for colonization against pathogenic fungus and the highest percentages of inhibition belonging to Talaromyces flavus 60 and Talaromyces flavus 136 were 59.52 and $57.61 \%$, respectively. Microscopic investigations showed that in regions where antagonistic isolates and Gaeumannomyces graminis var. tritici coincide, hyphal contact, penetration and fragmentation of Gaeumannomyces graminis var. tritici were observed. Investigating the effect of volatile and non-volatile compounds at $10 \mathrm{ml}$ concentration showed that the highest inhibition percentage on mycelium growth of the pathogen caused by $T$. harzianum (44.76\%) and T. longibrachiatum (52.38\%) respectively.
\end{abstract}

${ }^{*}$ Corresponding Author:

E-mail: mohammadi.myco@gmail.com

\section{Introduction}

Wheat forms one-fourth of world cereal production and is the main calorie source of over 1.5 milliard people. On average, wheat provides one-fifth of the calorie required for the whole people on the earth (FAO, 2003). Wheat take-all is caused by Gaeumannomyces graminis var. tritici. This root disease has been reported from most of the wheat cultivations all over the world (Huber and McCay-Buis, 1993). Fahima and Henis (1997) listed the pathogenic fungus among the eight important pathogenic fungi of wheat in the world and introduced it as one of the most important wheat pathogens in Oceania. Take-all disease control in infected area was to use herbicide, fungicide, soil improvement, burning plan residues, nutrition management and crop rotation (Yarham, 1981). Use of fungicides for the control of soil borne diseases is costly and also they have undesirable effects on environment, humankind and beneficial microorganisms in soil (Dluzniewska, 2003). This has diverted the attention of plant pathologists towards alternative methods for the control of plant diseases. Biological control using antagonistic fungi and bacteria is one of the proper practices to control the disease (Maghsoodloo et al. 2009). For example, Duffy et al. (1996) observed that combination of fluorescent Pseudomonads and Trichoderma koningii is more effective to biologically control take-all disease than a combination of several bacterial isolates (Duffy et al. 1996). Also, Kucuk and Kivanc (2003) reported that ten T. harzianum isolates inhibited in vitro growth of the pathogenic fungus mycelium from 28 to $100 \%$ (Kucuk and Kivanc, 2003). In a study the isolates of Talaromyces flavus isolated from cotton farms in Gorgan were tested to control cotton Verticillium wilt in vitro and in greenhouse conditions (Naraghi et al., 2007). They showed that the tested isolates were effective for the disease control and increased the yield as well. In a greenhouse research by Mehrabi Koshki et al. (2008) the useful effect of five $T$. virens isolates and two T. koningiopsis and T. viridescens isolates and a $T$. koningii isolate as well as biological compounds including trichodermin and subtilin on the control of take-all disease was reported. Moreover, in a study it was found that antagonistic isolates including $T$. harzianum B-1 and $T$. harzianum G-4 as well as fungicides like rural TS, propiconazole, benomyl, and carboxin thiram had high effect against take-all disease (Foroutan, 2008). This study was carried out to investigate the in vitro antagonistic effects of Trichoderma spp. and Talaromyces flavus isolates against Gaeumannomyces graminis var. tritici (Ggt), the causal agent of wheat take-all disease in 2011-2012. 


\section{Materials and methods}

Preparation of Biocontrol Agents

Trichoderma species used here were isolated by Iranian Pistachio Research Institute, Rafsanjan. Trichoderma species were grown on PDA media for further use.

\section{Preparation of Pathogenic Fungus}

Isolate T139 of Gaeumannomyces graminis var. tritici was provided by Zarghan Agriculture Research Center in Fars province, Iran.

\section{Pathogen Preservation}

To preserve the pathogen, PDA blocks were isolated from margins of 4-day old colonies of the pathogenic fungus and transferred into laboratory tubes containing PDA medium. These tubes were kept at $25^{\circ} \mathrm{C}$ for one week. Then the cotton on top of the tubes was flamed and the tubes were sealed using parafilm and stored at $7^{\circ} \mathrm{C}$.

Determination of the in Vitro Antagonistic Activities of Biocontrol Agents

In order to investigate the ability of Trichoderma and Talaromyces flavus isolates for inhibition of mycelium growth, establishment and development of Gaeumannomyces graminis var. tritici, the causal agent of wheat take-all disease, and also to investigate if the antagonism is due to parasitism or antibiosis, the following experiments were conducted:

Dual culture test: This was carried out to compare the ability of different Trichoderma and Talaromyces flavus isolates for in vitro growth inhibition of Ggt and also their establishment and development on the pathogenic fungus. In dual culture, 5-mm rings from the margins of 4-day old colonies of the pathogenic fungus were placed on one side of 9-cm Petri dishes containing PDA and on the opposite side placed the 5-mm ring from the margins of 4-day old colonies belonging to $T$. koningii, $T$. harzianum $\mathrm{S}, T$. harzianum $\mathrm{M}$, T. harzianum A, T. harzianum $\mathrm{D}, T$. virens, T. koningii, Talaromyces flavus 134, Talaromyces flavus 136, Talaromyces flavus 75, Talaromyces flavus 60 and $T$. longibrachiatum with $1 \mathrm{~cm}$ margins (Mohammadi et al. 2010). A 5-mm ring from the margins of pathogenic fungus was placed at the center of a plate as control. Petri dishes were kept at $25^{\circ} \mathrm{C}$ and diametrical growth measurements were carried out for three times with 24,72 and $120 \mathrm{hr}$ intervals after incubation. The inhibitory percentage of mycelium growth was determined using the Abbott's formula (Abbott, 1925):

$$
\text { MGIP }=\frac{\mathrm{CDGC}-\mathrm{CDGT}}{\mathrm{CDGC}} \times 100
$$

MGIP: mycelium growth inhibitory percentage

CDGC: colony diametrical growth of control

CDGT: colony diametrical growth of treatment

In this experiment in addition to mycelium growth inhibitory percentage of different Trichoderma and Talaromyces flavus isolates, against the pathogenic fungus after the growth was halted, development on takeall agent was investigated as well.
Direct contact:

i) By microscope slide: Sterile microscope slides were placed at the center of Petri dishes $(10 \mathrm{~cm})$ containing PDA media. A mycelial disc $(5 \mathrm{~mm})$ from the margin of 4day old colonies of Gaeumannomyces graminis var. tritici were placed each one side of each Petri dish and a mycelial disc $(5 \mathrm{~mm})$ from margin of 4-day colonies of Trichoderma and Talaromyces flavus isolates were placed on the other side. For each isolate, three replicates were considered. Petri dishes incubated at $25^{\circ} \mathrm{C}$ for 7 days. Then microscope slides were removed from the media, their bottom side were cleaned and effect and interactions among fungi was investigated under the microscope with different magnifications (Mohammadi et al. 2010).

ii) By creation stria: Strip with one centimeter width was cut by a sterile scalpel at the center of the petri dishes $(10 \mathrm{~cm})$ containing the PDA media. A mycelial disc $(5 \mathrm{~mm})$ from the margin of 4-day old colonies of Gaeumannomyces graminis var. tritici were placed one side of each Petri dish and a mycelial disc $(5 \mathrm{~mm})$ from margin of 4-day old colonies of Trichoderma and Talaromyces flavus isolates were placed on the other side. After 5 days pathogenic and antagonistic isolates collided. Then hyphal contact and connection were investigated under the microscope (Mohammadi et al. 2010).

The effect of volatile compounds: Effect of volatile metabolites produced by Trichoderma and Talaromyces flavus isolates against test pathogen were evaluated by the method of Dennis and Webster (1971). The mycelia disc (5mm) from margins of 4-day colonies of Trichoderma and Talaromyces flavus isolates were placed on center of a 10-cm Petri dishes. A 5-mm ring from PDA was placed instead of 5-mm ring of antagonistic isolates the center of control plates. After $24 \mathrm{~h}$, the top of each Petri dish was replaced with the bottom of Gaeumannomyces graminis var. tritici plates, so as test pathogen was directly exposed to the antagonistic environment created by antagonistic isolates. The pairs of each Petri dish were fixed and sealed together with paraffin tape and incubated at $25^{\circ} \mathrm{C}$. Radial growth of the pathogen was recorded after 24,72 and $120 \mathrm{~h}$ of incubation and percentage inhibition of pathogenic mycelium growth was calculated by Abbott's formula (section 2.4.1).

The effect of non-volatile compounds : This test was conducted to investigate the effects of non-volatile compounds produced by Trichoderma and Talaromyces flavus isolates against the mycelial growth of the pathogenic fungus. Four $1-\mathrm{cm}$ disks from the margins of Trichoderma colonies were transferred into 250-ml flasks containing PDB medium and four $1-\mathrm{cm}$ disks from Talaromyces flavus isolates were also transferred into 250-ml flasks containing liquid Czapek Dox Broth medium. The flasks were shaken using a rotary shakeroven at $25^{\circ} \mathrm{C}$ with 120 shake/min for Trichoderma isolates, and 50 shake/min for Talaromyces flavus isolates. After 10 days the contents of the flasks were removed and extracted using autoclaved millipore and vacuum pump with $0.22-\mu \mathrm{m}$ microfilters. The resulting solution was used to investigate the effects of liquid exudates from Trichoderma and Talaromyces flavus isolates on the mycelial growth of the pathogenic fungus. 
In this experiment for each Trichoderma and Talaromyces flavus isolate two 5- and 10-ml treatments from the extract were added to $20 \mathrm{ml}$ of PDA medium at room temperature and mixed well before transferring into the plates. When the plates were cooled enough, a 5-mm ring from the margins of 4-day colonies belonging to take-all pathogen was placed on the center of each plate and the plates were kept at $25^{\circ} \mathrm{C}$ (Mohammadi et al. 2010).

The diametrical growth of pathogenic fungus was measured at 24, 72 and $120 \mathrm{hr}$ after incubation. The percentage inhibition of mycelium growth was determined using the above formula.

\section{Statistical Analysis}

Dual culture test and investigation of volatile compound effects were performed in a completely randomized design consisting of 12 treatments with three replicates. The data from the experiments were statistically analyzed. The treatments were compared at $p \leq 0.01$ using Duncan multiple range test (Little and Hills, 1978).

\section{Results}

\section{Dual Culture Test}

All tested isolates were successful to inhibit the growth of pathogen and the isolates including Talaromyces flavus 60, Talaromyces flavus 134, Talaromyces flavus 136, T. harzianum A and $T$. harzianum $\mathrm{S}$ covered the pathogen colonies and became dominant. Thus Trichoderma and Talaromyces flavus isolates completely covered the pathogen colonies after 34 and 6 days, respectively.

Statistical analysis of the data showed that there is a significant difference between inhibitory effects of Trichoderma and Talaromyces flavus isolates $(\mathrm{P} \leq 0.01)$.

When the percentage of inhibition from Trichoderma and Talaromyces flavus isolates were compared it was determined that the highest levels of inhibition were achieved by using Talaromyces flavus 60 and Talaromyces flavus 136 after $120 \mathrm{hr}$ (59.52 and 57.61\%, respectively). No significant difference was observed between Talaromyces flavus 60 and Talaromyces flavus 136 (Table 1).

\section{Direct Contact}

The microscopic investigations showed that at earlier stages of contact, the hyphae of all tested antagonistic isolates contacted with the hyphae of the fungal pathogen in some regions and grew paralel to them. As the time passed the hyphal contact increased and direct penetration of antagonistic hyphae into the fungus hyphae was also observed. While using groove method, fragmentation was also observed in case of Talaromyces flavus 134. However, none of the antagonistic isolates formed hyphal coil.

\section{The Effects of Volatile Compounds}

There was a significant difference $(\mathrm{P} \leq 0.01)$ between Trichoderma and Talaromyces flavus isolates in terms of inhibiting the mycelium growth of Gaeumannomyces graminis var. tritici.

When the mean values of mycelium growth inhibitory effects on Ggt due to volatile compounds of Trichoderma and Talaromyces flavus isolates were compared, it was shown that the highest level of inhibition $(44.76 \%)$ caused by $T$. harzianum after $120 \mathrm{hr}$. As time passed, the percentage of inhibition by $T$. harzianum increased. In case of other isolates this trend was decreasing from 72 to $120 \mathrm{hr}$ (Table 2).

\section{The Effect of Non-Volatile Compounds}

The results from the statistical analysis showed that there was significant difference among Trichoderma and Talaromyces flavus isolates in terms of the effect of nonvolatile compounds ( 25 and $50 \%$ ) on the inhibition of the mycelial growth of Ggt.

When the means of inhibition rates of non-volatile compounds (25\%) from Trichoderma and Talaromyces flavus isolates were compared, it was found that the highest levels of inhibition caused by Talaromyces flavus 136, T. harzianum and T. harzianum $\mathrm{S}$ as 51.41, 40.88 and $37.73 \%$, respectively, after $72 \mathrm{hr}$. No significant difference was found between Talaromyces flavus 136, T. harzianum and $T$. harzianum S. Also, when the percentages of mycelium growth inhibition of Ggt by non-volatile compounds $(50 \%)$ of Trichoderma and Talaromyces flavus isolates were compared, it was determined that $T$. longibrachiatum caused the highest inhibition rates of 52.83 and $52.38 \%$ at two recording intervals. In case of $T$. harzianum $\mathrm{M}$ and $T$. koningi the inhibition percentage increased as time passed while about other isolates this trend was first increasing from 24 to $72 \mathrm{hr}$ and then decreasing from 72 to $120 \mathrm{hr}$ (Table 3, 4).

Table 1 The mean percentage inhibition of the colony growth of wheat take-all agent Gaeumannomyces graminis var. tritici by different treatments, 24,72 and 120 hours after incubation

\begin{tabular}{l|lll}
\hline Treatment & Mean $(24 \mathrm{~h})$ & Mean $(72 \mathrm{~h})$ & Mean $(120 \mathrm{~h})$ \\
\hline Talaromyces flavus 60 & $52.22 \mathrm{a}$ & $52.28 \mathrm{a}$ & $59.52 \mathrm{a}$ \\
Talaromyces flavus 136 & $51.11 \mathrm{a}$ & $52.28 \mathrm{a}$ & $57.61 \mathrm{a}$ \\
Trichoderma harzianum S & $51.11 \mathrm{a}$ & $50.32 \mathrm{ab}$ & $49.04 \mathrm{~b}$ \\
Trichoderma harzianum A & $48.89 \mathrm{a}$ & $45.75 \mathrm{~b}$ & $46.43 \mathrm{bc}$ \\
Talaromyces flavus 134 & $45.55 \mathrm{a}$ & $39.21 \mathrm{c}$ & $44.28 \mathrm{c}$ \\
Trichoderma harzianum & $20.00 \mathrm{~b}$ & $35.94 \mathrm{~cd}$ & $43.81 \mathrm{c}$ \\
Trichoderma harzianum M & $16.66 \mathrm{bc}$ & $34.63 \mathrm{cde}$ & $35.23 \mathrm{~d}$ \\
Trichoderma longibrachiatum & $14.44 \mathrm{bc}$ & $33.98 \mathrm{cde}$ & $31.90 \mathrm{de}$ \\
Trichoderma koningi & $13.33 \mathrm{bc}$ & $33.33 \mathrm{de}$ & $29.04 \mathrm{ef}$ \\
Trichoderma virens & $12.22 \mathrm{bc}$ & $30.06 \mathrm{e}$ & $26.19 \mathrm{f}$ \\
Talaromyces flavus 75 & $8.89 \mathrm{c}$ & $20.26 \mathrm{f}$ & $21.43 \mathrm{~g}$ \\
\hline
\end{tabular}


Table 2 Mean rate of inhibition of the colony growth of wheat take-all agent Gaeumannomyces graminis var. tritici by the volatile metabolites of Trichoderma and Talaromyces flavus isolates, 24, 72 and 120 hours after incubation

\begin{tabular}{l|lll}
\hline Treatment & Mean $(24 \mathrm{~h})$ & Mean $(72 \mathrm{~h})$ & Mean $(120 \mathrm{~h})$ \\
\hline Trichoderma harzianum S & $53.57 \mathrm{a}$ & $57.74 \mathrm{a}$ & $40.95 \mathrm{~b}$ \\
Trichoderma koningi & $51.19 \mathrm{ab}$ & $55.95 \mathrm{a}$ & $29.04 \mathrm{~d}$ \\
Trichoderma longibrachiatum & $47.02 \mathrm{abc}$ & $45.24 \mathrm{~b}$ & $34.76 \mathrm{c}$ \\
Trichoderma harzianum & $43.45 \mathrm{abcd}$ & $38.09 \mathrm{~cd}$ & $44.76 \mathrm{a}$ \\
Trichoderma virens & $42.85 \mathrm{abcd}$ & $38.69 \mathrm{~cd}$ & $21.43 \mathrm{e}$ \\
Trichoderma harzianum M & $40.47 \mathrm{bcd}$ & $38.69 \mathrm{~cd}$ & $26.66 \mathrm{~d}$ \\
Talaromyces flavus 60 & $35.71 \mathrm{cde}$ & $48.81 \mathrm{~b}$ & $20.95 \mathrm{e}$ \\
Talaromyces flavus 75 & $34.52 \mathrm{cde}$ & $26.76 \mathrm{e}$ & $7.14 \mathrm{f}$ \\
Talaromyces flavus 134 & $32.14 \mathrm{de}$ & $34.52 \mathrm{de}$ & $21.90 \mathrm{e}$ \\
Talaromyces flavus 136 & $30.95 \mathrm{de}$ & $39.28 \mathrm{~cd}$ & $36.90 \mathrm{c}$ \\
Trichoderma harzianum A & $25 \mathrm{e}$ & $43.45 \mathrm{bc}$ & $36.66 \mathrm{c}$ \\
\hline
\end{tabular}

Table 3 Mean rates of inhibition of the colony growth of wheat take-all agent Gaeumannomyces graminis var. tritici by 25\% concentration of the non-volatile exudates of Trichoderma and Talaromyces flavus isolates, 24, 72 and 120 hours after incubation

\begin{tabular}{l|lll}
\hline Treatment & Mean $(24 \mathrm{~h})$ & Mean $(72 \mathrm{~h})$ & Mean $(120 \mathrm{~h})$ \\
\hline Talaromyces flavus 136 & $38.88 \mathrm{a}$ & $41.51 \mathrm{a}$ & $10.47 \mathrm{de}$ \\
Talaromyces flavus 134 & $34.44 \mathrm{ab}$ & $27.67 \mathrm{c}$ & $7.61 \mathrm{e}$ \\
Talaromyces flavus 75 & $31.11 \mathrm{abc}$ & $28.93 \mathrm{c}$ & $10.95 \mathrm{de}$ \\
Talaromyces flavus 60 & $25.55 \mathrm{abc}$ & $32.70 \mathrm{bc}$ & $8.09 \mathrm{e}$ \\
Trichoderma harzianum & $24.44 \mathrm{abc}$ & $40.88 \mathrm{a}$ & $8.09 \mathrm{e}$ \\
Trichoderma longibrachiatum & $23.33 \mathrm{abc}$ & $30.19 \mathrm{bc}$ & $23.09 \mathrm{a}$ \\
Trichoderma harzianum S & $22.22 \mathrm{abc}$ & $37.73 \mathrm{ab}$ & $11.90 \mathrm{~cd}$ \\
Trichoderma harzianum A & $20 \mathrm{bc}$ & $28.93 \mathrm{c}$ & $20.95 \mathrm{a}$ \\
Trichoderma harzianum M & $18.89 \mathrm{bc}$ & $32.07 \mathrm{bc}$ & $15.71 \mathrm{~b}$ \\
Trichoderma koningi & $16.67 \mathrm{c}$ & $31.44 \mathrm{bc}$ & $20.47 \mathrm{a}$ \\
Trichoderma virens & $15.55 \mathrm{c}$ & $34.59 \mathrm{bc}$ & $14.76 \mathrm{bc}$ \\
\hline
\end{tabular}

Table 4 Mean rates of inhibition of the colony growth of wheat take-all agent Gaeumannomyces graminis var. tritici by $50 \%$ concentration of the non-volatile exudates of Trichoderma and Talaromyces flavus isolates, 24, 72 and 120 hours after incubation

\begin{tabular}{l|lll}
\hline Treatment & Mean $(24 \mathrm{~h})$ & Mean $(72 \mathrm{~h})$ & Mean $(120 \mathrm{~h})$ \\
\hline Trichoderma harzianum A & $43.33 \mathrm{a}$ & $50.94 \mathrm{a}$ & $32.38 \mathrm{~cd}$ \\
Trichoderma longibrachiatum & $41.11 \mathrm{ab}$ & $52.83 \mathrm{a}$ & $52.38 \mathrm{a}$ \\
Talaromyces flavus 75 & $36.66 \mathrm{abc}$ & $42.77 \mathrm{~b}$ & $15.23 \mathrm{fg}$ \\
Talaromyces flavus 136 & $34.44 \mathrm{abcd}$ & $33.33 \mathrm{de}$ & $29.04 \mathrm{~d}$ \\
Trichoderma harzianum S & $28.89 \mathrm{bcde}$ & $35.22 \mathrm{cde}$ & $23.81 \mathrm{e}$ \\
Trichoderma virens & $23.33 \mathrm{cdef}$ & $41.51 \mathrm{bc}$ & $30 \mathrm{~d}$ \\
Trichoderma koningi & $22.22 \mathrm{def}$ & $34.59 \mathrm{cde}$ & $35.23 \mathrm{c}$ \\
Trichoderma harzianum & $22.22 \mathrm{def}$ & $40.88 \mathrm{bc}$ & $18.09 \mathrm{f}$ \\
Trichoderma harzianum $\mathrm{M}$ & $17.78 \mathrm{efg}$ & $40.25 \mathrm{bcd}$ & $42.38 \mathrm{~b}$ \\
Talaromyces flavus 134 & $12.22 \mathrm{fg}$ & $30.81 \mathrm{e}$ & $14.28 \mathrm{fg}$ \\
Talaromyces flavus 60 & $5.55 \mathrm{~g}$ & $39.62 \mathrm{bcd}$ & $12.38 \mathrm{~g}$ \\
\hline
\end{tabular}

\section{Discussion}

Trichoderma is one of the biocontrol agents of take-all pathogenic fungus whose control mechanisms are competition, mycoparasitism, the function of non-volatile compounds and volatile compounds. These mechanisms were previously investigated and confirmed by other researchers such as Arianpour (2011) and Mehrabi Koushki et al. (2008). The important fact which was pointed out by many researchers is the presence of difference among species and special various isolates of a

species in terms of control mechanisms and the intensity of them which is consistent with the present study. The fungus Talaromyces flavus is the biocontrol agent of several soil-borne pathogens whose isolates were not yet evaluated against take-all pathogenic fungus. The present research evaluated the antagonistic effects of seven Trichoderma isolates and four Talaromyces flavus isolates which were previously applied for biological control of other pathogenic agents, against G. graminis 
var. tiritici.

Macroscopic investigations about the contact of Trichoderma and Talaromyces flavus isolates using dual culture showed that all tested isolates inhibited the growth of the pathogenic fungus as rapid growth of Trichoderma provided competitive conditions for place (medium) and food. The study by Kucuk and Kivanc (2003) and Mehrabi Koushki et al. (2008) confirmed these results. The isolates $T$. harzianum A, T. harzianum $\mathrm{S}$, Talaromyces flavus 134, Talaromyces flavus 136 and Talaromyces flavus 60 were able to colonize the mycelium of the pathogenic fungus. The same results were reported by Mehrabi Koushki et al. (2008) and Naraghi et al. (2007). Hermosa et al. (2000) placed some pathogenic fungi against some isolates of Trichoderma and concluded that sporulation and growth of Trichoderma on colonies of the pathogenic fungi depend on the type of pathogen and combination of medium. The highest level of growth inhibition caused by Talaromyces flavus 60 and Talaromyces flavus 136 and it was found that these isolates have higher competitive potential against the take-all pathogenic fungus than other isolates. The results from Naraghi et al. (2010c) reflect high feeding ability of Talaromyces flavus.

Microscopic investigation on region where antagonistic Trichoderma and Talaromyces flavus isolates coincide with the take-all pathogenic fungus showed that hyphal penetration, contact and fragmentation occurred. This was consistent with the results of Naraghi et al. (2010a, 2010b and 2010c) and Heidari Faroughi et al. (2005). The hyphae of antagonistic isolates have positive tropism towards those of the pathogenic fungus. This tropism may be due to chemical compounds on the cell wall of the pathogenic fungus hyphae. The parasitism mechanism of Trichoderma is complicated and includes chemical tropism, identification of lactine presented on the cell wall of the pathogen (Inbar and Chet, 1995) and formation of appressorium, penetrating organs and pathogen-trapping rings (Elad and Chet 1983).

To investigate the antibiosis, two tests were carried out for evaluating the effects of volatile and non-volatile exudates from Trichoderma and Talaromyces flavus isolates against Ggt. These tests revealed that various Trichoderma and Talaromyces flavus isolates are different in terms of producing volatile and non-volatile compounds which inhibit the growth of host fungus. Due to this, some Trichoderma isolates were more effective to inhibit the growth of the pathogenic fungus. Our results were consistent with the results achieved by Iraqi et al. (2008). Generally, the results show that various Trichoderma species and even the different isolates of a species produce variety of volatile materials which have different effects on various fungi. These isolates may produce a series of volatile metabolites with different amounts or may produce completely different chemical compounds. The investigations have shown that $T$. harzianum produces a wide range of antibiotics and enzymes against various fungi. Volatile and non-volatile metabolites can disperse throughout soil pores so that there is no need for direct contact with the disease agent to affect. With respect to the results, it is shown that species and even isolates of a species act differently in terms of antagonistic mechanisms for biocontrol of the pathogenic agent (Lorito et al., 1994).

The results from investigating the effect of nonvolatile exudates from Trichoderma and Talaromyces flavus isolates were consistent with the results reported by Iraqi et al. (2008). Moreover, Hashemi et al. (2012) reported the same results when he tested the effect of nonvolatile exudates on prevented growth of Fusarium oxysporum f. sp. sesame.

Most Trichoderma strains produce toxic volatile and non-volatile anti-fungal compounds which halt the growth of pathogenic fungi (Vey et al., 2001). In another study, the role of Trichoderma spp. to produce antibiotics such as trichodermin, trichodermol, harzianum $\mathrm{A}$ and harizanulid was pointed out (Kucuk and Kivanc, 2004).

Experimental investigations are proper methods to elementary identification of antagonistic microorganisms. However, the usefulness of an antagonist cannot be validated only based on experimental data as the effect of antagonist directly against the pathogenic agent is mostly tested on rich medium while the effect of microorganisms in natural environment is highly affected by many factors such as temperature, $\mathrm{pH}$, humidity, soil texture and the behavior of other microorganisms. For example, an isolate may be effective against a pathogen in vitro while it may fail to control the pathogen in natural environment due to the competition with other antagonists. Due to this, it is recommended that all in vitro tested species are investigated in greenhouse conditions as well. According to danger of fungicides these antagonistic isolates can be used as agents for the control of plant disease after in vivo trials.

\section{Acknowledgments}

The authors gratefully acknowledge the gift of biocontrol isolates from Dr. A.H. Mohammadi (Iranian Pistachio Research Institute, Rafsanjan) and providing fungal pathogen of wheat take-all from Dr. A. Fasihiani (Agricultural and Natural Resources Research Center of Fars, Zarghan).

\section{References}

Abbott WS. 1925. A method of computing the effectiveness of insecticide. J. Econ. Ent. 18: 265-267.

Arianpour A. 2011. Investigate the possibility of biocontrol of wheat take-all disease by Trichoderma isolates and Pseoudomonas fluoroescens. Yasouj. University of Yasouj.

Dennis C, Webster J. 1971. Antagonism properties of species groups of Trichoderma. Trans. Br. Mycol. Soc. 57: 363-369.

Dluzniewska J. 2003. Reaction of fungi of Trichoderma genus to selected abiotic factors. Elec. J. Polish Agr. Uni. Agron. 6: 4-8.

Duffy BK, Simon A, Weller DM. 1996. Combination of Trichoderma koningii with fluorescent pseudomonads for control of take-all on wheat. Phytopathol. 86: 188-194.

Elad Y, Chet I. 1983. Improved selective media for isolation of Trichoderma spp. or Fusarium spp. Phytoparasitica. 11: 55-58.

Fahima T, Henis Y. 1997. Increasing of Trichoderma hamatum and Talaromyces flavus on the Root of safe and unsafe Hosts. Tehran: Research, Education and Extension organization. 085198-637-4. 
FAO: 2003. Food and Agriculture Organization of the United Nations; [cited 2003 Feb 25]. Available from: http:// www.FAO.org/.

Foroutan A. 2008. Increasing the efficiency of strains Trichoderma harzianum against wheat take all disease by adding seed disinfectant fungicides. In: 18th Iranian Plant Protection Congress. Hamedan, 24-27 Jul. Iran. pp: 400.

Hashemi SL, Mohammadi S, Basirnia T. 2012. Investigating biological contorl of Fusarium oxysporum f.sp. sesami causing wilt and yellowing in Sesamum indicum by various species of Trichoderma in vitro. In: 6th National Confpp:erence on New Ideas in Agriculture. Isfahan, 26-27 Oct. pp: 215.

Heidari Faroughi SH, Etebarian HR, Zamanizadeh HR. 2005. Evaluation of Trichoderma isolates for biological control of Phytophthora drechsleri in glasshouse. J. Appl. Ent. Phytopath. 72: 113-134

Hermosa MR, Grondona I, Iturriaga EA, Diaz-Minguez JM, Castro C, Monte E, Garcia-Acha I. 2000. Molecular Characterization and Identification of Biocontrol Isolates of Trichoderma spp. Applied and Environmental Microbiology, 66: 1890-1898.

Huber DM, McCay-Buis TS. 1993. A multiple component analysis of the take-all disease of cereals. Plant Disease, 77: 437-447.

Inbar J, Chet I. 1995. The role of recognition in the induction of specific chitinases during mycoparasitism by Trichoderma harzianum. J. Microb. 141: 2823-2829.

Iraqi MM, Rahnama K, Zafari D, Taghinasab M. 2008. Investigating biological control of Ophiostoma novo-ulmi, causal agent of Dutch Elm Disease by Trichoderma harzianum and T. virens in vitro. J. Agric. Sci. Natur. Resour. 14: 12-20.

Kucuk C, Kivanc M. 2003. Isolation of Trichoderma spp. and determination of their antifungal, biochemical and physiological features. Turk. J. Biol. 27: 247-253.

Kucuk C, Kivanc M. 2004. In vitro antifungal activity of strains of Trichoderma harzianum. Turk. J. Biol. 28: 111-115.

Little TM, Hills FJ. 1978. Agricultural experimentation design and analysis. New York: John Willey and Sons, Inc. 978-0-47102352-4.
Lorito M, Peterbauer C, Hayes CK, Harman GE. 1994. Synergistic interaction between fungal cell wall degrading enzymes and different antifungal compounds enhances inhibition of spore germination. J. Microb. 140: 623-629.

Maghsoodloo R, Ghorbani nasrabadi RS, Razavi A, Ebrahimi T. 2009. Evaluation of antagonistic bacteria strains Azotobacter against take-all agent under laboratory conditions. In: Paper 18th Iranian Plant Protection. hamedan, 24-27 Jul. Iran. pp: 389.

Mehrabi Koushki M, Zafari D, Rouhani H, Ghalandar M. 2008. Evaluation the effect of Trichoderma isolates, mustard flour and two commercial biological products in control of wheat take-all disease. J. Agri. Sci. 17: 197-208.

Mohammadi S, Mansoori B, Zamani Zadeh HR. 2010. Antagonistic Mechanisms of Trichoderma spp. against Rhizoctonia solani, the causal agent of chickpea wet root rot disease. Plant Protect. J. 1: 71-85.

Naraghi L, Heydari A, Rezaee S, Razavi M, Afshari-Azad H. 2010a. Biological control of greenhouse cucumber Verticillium wilt disease by Talaromyces flavus. Phytopathol. Mediterr. 49: 321329.

Naraghi L, Heydari A, Rezaee S, Razavi M, Jahanifar H, Mahmoodi Khaledi E. 2010b. Biological control of tomato Verticillium wilt disease by Talaromyces flavus. J. Plant Prot Res. 50: 360-365.

Naraghi L, Heydari A, Rezaee S, Razavi M, Jahanifar H. 2010c. Study on antagonistic effects of Talaromyces flavus on Verticillium albo-atrum, the causal agent of potato wilt disease. J. Crop. Protection. 29: 658-662.

Naraghi L, Zareh-Maivan H, Heydari A, Afshari-Azad H. 2007. Investigation of the effect of heating, vesicular arbuscular mycorriza and thermophilic fungs on cotton wilt disease. Pak. J. Biol. Sci. 10: 1596-1603.

Vey A, Hoag IRE, Butt TM. 2001. Toxic metabolites of fungal biocontrol agents. In: Butt Jackson C. and Magan N. Fungi as biocontrol agents: Progress, problems and potential. Bristol: CAB International. pp 311-346. 0-87893-403-0.

Yarham DJ. 1981. Practical Aspects of Epidemiology and Control. In: Asher MJC, Shipton PJ. Biology and Control of Take-all. London: Academic Press; p. 353-384. 0-660-13422-5 1. 